\title{
Differential Localization of Four Subspecies of Protein Kinase C in the Rat Striatum and Substantia Nigra
}

\author{
Chika Yoshihara, Naoaki Saito, Kohtaro Taniyama, ${ }^{a}$ and Chikako Tanaka \\ Department of Pharmacology, Kobe University School of Medicine, Kobe 650, Japan
}

The distribution of protein kinase $\mathrm{C}$ (PKC) subspecies and their colocalization with neurotransmitters were examined in the rat striatum and substantia nigra (SN), using immunocytochemistry. The $\alpha$ - and $\beta$ I-PKC immunoreactivies were seen predominantly in the perikarya of the neurons in the striatum and SN. In contrast, the $\beta$ II- and $\gamma$-PKC immunoreactivities were abundant in both the perikarya and the neuropils in the striatum and only in the neuropils in the SN.

From electron microscopic studies, the $\alpha$ - and $\beta \mathrm{I}-\mathrm{PKC}$ immunoreactivities were seen adjacent to the plasma membrane, while the $\beta$ II-PKC immunoreactivity was observed in the cytoplasm around the Golgi complex. The $\gamma$-PKC immunoreaction was dense throughout the cytoplasm.

The double-staining and lesion studies revealed that the $\alpha$-PKC-immunopositive neurons in the striatum were intrinsic cholinergic neurons, and that most of the $\alpha$-PKC-immunoreactive neurons in the $\mathrm{SN}$ were dopaminergic neurons. The $\beta$ I-PKC-immunoreactive neurons were intrinsic GABAergic neurons in the striatum. Moreover, most of the $\beta \| 1-$ and $\gamma$-PKC-immunoreactive neurons were medium-sized neurons projecting to the $\mathrm{SN}$, and over $90 \%$ of GABAergic neurons in the caudate-putamen contained $\beta \|-P K C$. The $\beta \|$ PKC-immunoreactive neurons showed no $\gamma$-PkC immunoreactivity, and the $\gamma$-PKC-immunoreactive neurons were not $\beta$ II-PKC immunoreactive.

These findings suggest that $\alpha$-PKC is related to the function of the nigral dopaminergic and the striatal cholinergic neurons, and that the $\beta \mathrm{I}-\mathrm{PKC}$ is involved in the function of the striatal intrinsic GABAergic neurons. The $\beta$ Il- and $\gamma$-PKC may also modulate a specific neuronal function in the striatonigral system.

Protein kinase $\mathrm{C}$ (PKC), a calcium-activated and phospholipiddependent protein kinase, has been considered to have a key role for cell-surface signal transduction (for reviews, see Nishizuka, 1984, 1986; Huang, 1990). Recent molecular cloning studies revealed that $\mathrm{PKC}$ is a large family consisting of at least 7 subspecies (Nishizuka, 1988). Early enzymological analysis revealed that $\mathrm{PKC}$ isolated from the rat brain can be resolved into

\footnotetext{
Received Aug. 7, 1990; revised Oct. 8, 1990; accepted Oct. 11, 1990.

This work was supported by research grants from the Scientific Research Fund of the Ministry of Education, Science, and Culture and the Ministry of Health and Welfare, Japan, from the Yamanouchi Foundation for Research on Metabolic Disorders, and from the Uehara Memorial Foundation. We thank M. Ohara for reading the manuscript.

Correspondence should be addressed to $C$. Tanaka at the above address.

aresent address: Second Department of Pharmacology, Nagasaki University School of Medicine, Nagasaki 852, Japan.

Copyright (c) 1991 Society for Neuroscience $0270-6474 / 91 / 110690-11 \$ 03.00 / 0$
}

3 major fractions (type I, type II, and type III) by hydroxyapatite-column chromatography (Huang et al., 1986), which correspond to $\gamma, \beta \mathrm{I}+\beta \mathrm{II}$, and $\alpha$, respectively (Kikkawa et al., 1987; Ono et al., 1987). The enzyme is widely distributed in various tissues and seems to be implicated in several physiological processes (Nishizuka, 1984, 1986). Among various tissues, the enzyme is most concentrated in the CNS (Minakuchi et al., 1981). Immunocytochemical and biochemical studies in this and other laboratories have revealed a differential regional and cellular localization of each PKC subspecies in the brain (Kitano et al., 1987; Hidaka et al., 1988; Huang et al., 1988; Kose et al., 1988; Saito et al., 1988, 1989; Hosoda et al., 1989; Ito et al., 1990). Particularly, the $\gamma$-PKC subspecies is found only in the CNS (Ohno et al., 1987).

Considerable numbers of phorbol ester binding sites, an indirect marker for PKC, were found in the striatonigral system (Worley et al., 1986). Immunocytochemical studies also showed a specific localization of the PKC subspecies in the striatum and substantia nigra (SN; Huang et al., 1987; Saito et al., 1988, 1989). Furthermore, we noted a marked decrease in phorbol ester binding sites in the striatum from patients with Parkinson's disease (Nishino et al., 1989) or Huntington's disease (Kitamura et al., 1990), a neurodegenerative disease of the nigrostriatal or striatonigral system, respectively. We now report detailed distributions of each PKC subspecies within the striatum and the $\mathrm{SN}$ and the colocalization with various neurotransmitters, findings that aid in elucidating the functions of each PKC subspecies in striatonigral and nigrostriatal systems.

\section{Materials and Methods}

Preparations of antibodies against PKC subspecies and various neurotransmitter markers. The rabbit antisera specific to the PKC subspecies were prepared against synthetic oligopeptides that correspond to the carboxyl-terminal portion of each subspecies (QFVHPILQSAV for the $\alpha$-PKC, SYTNPEFVINV for the $\beta$ I-PKC, and SFVNSEFLKPEVKS for the BII-PKC; Hosoda et al., 1989; Saito et al., 1989; Ito et al., 1990). The monoclonal antibody to $\gamma$-PKC, obtained by immunizing mice with the purified PKC from the soluble fraction of rat brain, was characterized (Hashimoto et al., 1988). The mouse polyclonal antisera specific to the $\alpha$ - and $\beta$ II-PKC were also obtained by immunizing mice with the same antigens used for the rabbit. Western blot analysis revealed that the antibodies reacted specifically with each subspecies.

The monoclonal antibody against GABA and the rabbit antiserum against GABA were raised and characterized, as described (Saito et al., 1986; Sakaue et al., 1989).

The rabbit antisera against CAT and tyrosine hydroxylase $(\mathrm{TH})$ were purchased from Chemicon and Eugene Tech International Inc., respectively.

Preparation of tissue sections. Male Wistar rats weighing $200 \mathrm{gm}$ were separated into 3 groups: the first group was used as controls, the second group was given an intraventricular injection of colchicine $(0.5 \mu \mathrm{g} / \mathrm{gm})$ $48 \mathrm{hr}$ before perfusion, and the third group was treated with a unilateral 
intracerebral injection of $50 \mathrm{~nm}$ kainic acid (in $20 \mu \mathrm{l}$ saline) given into the caudate-putamen (CP) $7 \mathrm{~d}$ before perfusion. The rats were anesthetized with an intraperitoneal injection of pentobarbital $(50 \mathrm{mg} / \mathrm{kg})$ and perfused through the left ventricle with saline, followed by fixatives. To obtain adequate staining of each antibody, 2 fixative solutions were prepared: fixative A, $0.5 \%$ glutaraldehyde, $4 \%$ paraformaldehyde, and $0.2 \%$ picric acid in $0.1 \mathrm{M}$ phosphate buffer $(\mathrm{pH}, 7.0$ ); and fixative $\mathrm{B}$, periodate-lysine-paraformaldehyde fixative containing $0.05 \%$ picric acid. Fixative $A$ was suitable for the immunostaining of the $\beta \mathrm{I}-, \beta \mathrm{II}-$, and $\gamma$-PKCs and GABA, and fixative B was proper for $\alpha$-PKC, TH, and CAT. After perfusion, the brain was removed from the cranial cavity, immersed in fixative $\mathrm{A}$ without glutaraldehyde or in fixative $\mathrm{B}$, and kept in $30 \%$ sucrose in $0.1 \mathrm{~m}$ phosphate buffer for several days. The brains were then frozen and sectioned frontally at $20-\mu \mathrm{m}$ thickness using a cryostat, for light microscopy. For electron microscopy, the brains were frozen in liquid nitrogen, thawed, and cut into $40-\mu \mathrm{m}$-thick frontal sections on a vibratome.

Immunocytochemical procedures for the PKC subspecies. The PBS used here contained $0.03 \%$ Triton X-100 for light microscopic immunocytochemistry but not for electron microscopy. The sections were preincubated with $0.3 \% \mathrm{H}_{2} \mathrm{O}_{2}$ and $5 \%$ normal goat serum in PBS containing $0.03 \%$ Triton X-100 (PBS-T) to block the endogenous peroxidase activity and nonspecific binding of the antibodies. The preparations were subsequently incubated with primary antibodies at appropriate concentrations in PBS-T containing 5\% normal goat serum for $18 \mathrm{hr}$ at $4^{\circ} \mathrm{C}$. After washing with PBS-T, the sections were incubated for an additional $4 \mathrm{hr}$ with goat anti-mouse IgG (ICN, Lisle, IL) or goat antirabbit IgG (MBL, Nagoya, Japan), then were incubated for $1.5 \mathrm{hr}$ with mousc peroxidase-antiperoxidase (PAP; ICN, Lisle, IL) complex or rabbit PAP complex (ICN, Lisle, IL). After 3 rinses, the preparations were developed with 0.02\% 3,3'-diaminobenzidine (Sigma), 0.2\% nickel ammonium sulfate, and $0.005 \% \mathrm{H}_{2} \mathrm{O}_{2}$ in $50 \mathrm{~mm}$ Tris- $\mathrm{HCl}(\mathrm{pH}, 7.4)$. The sections were observed and photographed under a Zeiss light microscope.

For electron microscopy, the immunostained sections were washed in PBS, postfixed for $1 \mathrm{hr}$ in $2 \%$ osmium tetroxide in $0.1 \mathrm{~m}$ PBS, dehydrated in a graded series of ethanol, and then flat embedded on siliconized slides in Epon. After polymerization at $60^{\circ} \mathrm{C}$ for $48 \mathrm{hr}$, the selected areas were cut off and attached to Epon supports for further sectioning on a Reichert-Jung Ultracut E ultramicrotome. Ultrathin sections were cut and mounted on 200-mesh uncoated grids (MAXTAFORM), counterstained with $1 \%$ uranyl acetate in $50 \%$ ethanol, and cxamincd with a JEM $100 \mathrm{SX}$ clectron microscope.

Immunocytochemical procedures for double labeling. We used doublelabeling methods to examine which transmitters coexist with each of the PKC subspecies. The sections for double labeling were first stained with antibody against one of the PKC subspecies by the PAP method, as described above. The stained sections already labeled with the dark purple products for the PKC subspecies were then incubated with antibodies to neurotransmitter markers (GABA, CAT, TH) or other PKC subspecies for $18 \mathrm{hr}$ at $4^{\circ} \mathrm{C}$, followed by fluorescein isothiocyanate (FITC)conjugated goat anti-rabbit IgG or goat anti-mouse IgG Fab antibodies for $4 \mathrm{hr}$ at room temperature. The sections were then mounted on slides with buffered glycerol for fluorescent or light microscopic observation.

\section{Results}

PKC-subspecies in the caudate-putamen and globus pallidus

\section{Light and electron microscopic localization of $P K C$ subspecies}

As illustrated in Figure 1, $A$ and $E$, the antisera to $\alpha$-PKC labeled the large, scattered neurons (40-45 $\mu \mathrm{m}$ in diameter) in the $C P$ and globus pallidus (GP). The immunoreactive neurons had a moderate immunoreaction in the perikaryal and proximal dendritic cytoplasm, and the perinuclear coarse, immunopositive dots were also seen in the perikarya. There was a diffuse immunoreactivity in the neuropils of the $\mathrm{CP}$ and a rather weak immunoreactivity in those of the GP. The fiber bundles were immunonegative.

The $\beta \mathrm{I}$-PKC immunoreactivity was found in medium to large neurons (25-28 $\mu \mathrm{m}$ in diameter) scattered evenly in the gray matter of the CP (Fig. $1 B, F$ ). The immunoreactivity was present homogeneously in the periphery of the perikarya and in the proximal part of the dendrites. The neuropils and fiber bundles showed very weak immunoreactivity.

The $\beta$ II-PKC immunoreactivity was seen in many mediumsized neurons (approximately $20 \mu \mathrm{m}$ in diameter) throughout the CP and GP, predominantly in the caudal part. The immunoreactive products could be seen in a dotted pattern in the perinuclear area (Fig. $1 C, G$ ). The neuropils in both the $\mathrm{CP}$ and the GP showed intense immunoreactivity, which was more intense in the CP than in the GP. The fiber bundles were weakly immunopositive.

The $\gamma$-PKC immunoreactivity was found in numerous medium-sized neurons (18-22 $\mu \mathrm{m}$ in diameter) scattered throughout the CP and GP. The immunoreactivity was dense in the perikarya and the proximal part of dendrites, but light in the nuclei (Fig. $1 D, H)$. In addition, the immunoreaction was observed in the neuropils and fiber bundles.

Electron microscopic analysis of the perikarya of the immunoreactive neurons for each PKC subspecies showed findings as follows: the $\alpha$-PKC immunoreactivity was present in the fringe of the perikaryon exhibiting the immunoreactive band along the plasma membrane, and small clusters of the immunoreaction were also scattered sparsely in the perinuclear area (Fig. $2 A$ ). The $\beta \mathrm{I}-\mathrm{PKC}$ immunoreactivity was seen in the perikaryon just adjacent to the plasma membrane, and the fringe staining consisted of the clustered immunoreaction (Fig. $2 B$ ). The $\beta$ II-PKC immunoreactivity was observed in the cytoplasm

Figure 1. Light microscopic localization of PKC subspecies in rat CP and GP. $A-D$, Lower magnification: scale bars, $250 \mu \mathrm{m}$. $E-H$, Higher magnification: scale bars, $30 \mu \mathrm{m}$. $A$ and $E$, The $\alpha$-PKC immunoreactivity is distributed in the scattered large neurons and the neuropils. The immunopositive neurons show a moderate immunoreaction along the perikaryal and proximal dendritic membrane and show dense immunopositive dots in the perikarya. $B$ and $F$, The $\beta \mathrm{I}$-PKC immunoreactivity is seen predominantly in the scattered medium to large neurons. The immunoreaction is present homogeneously within the perikarya and in the proximal part of the dendrites. $C$ and $G$, The $\beta$ II-PKC immunoreactivity is observed in the numerous medium-sized neurons and in the neuropils. The immunoreactive products could be seen in a dotted pattern in the perinuclear area. $D$ and $H$, The $\gamma$-PKC immunoreactivity is observed in many medium-sized neurons and the neuropils. The immunomaterials are dense in the perikarya and the proximal part of dendrites, but light in the nuclei.

Figure 2. Electron micrographs of neuronal perikarya showing immunoreactivity of PKC subspecies in CP. $A$, The $\alpha$-PKC immunoreactivity is present in the fringe of the perikaryon exhibiting an immunoreactive band along the plasma membrane, and small clusters of the immunoreaction are scattered sparsely in the perinuclear area. $B$, The $\beta \mathrm{I}-\mathrm{PKC}$ immunoreactivity is seen in the perikaryon just adjacent to the plasma membrane, and the fringe staining consisted of a clustered immunoreaction (arrows). $C$, The $\beta$ III-PKC immunoreactivity is observed in the cytoplasm around the concave face (trans region) of the Golgi complex and on its membrane (arrows). $D$, the $\gamma$-PKC immunoreaction is dense throughout the perikaryal cytoplasm, except for the interior of the cell organelles, and is light in the nucleoplasm. Scale bars, $1 \mu \mathrm{m}$. 


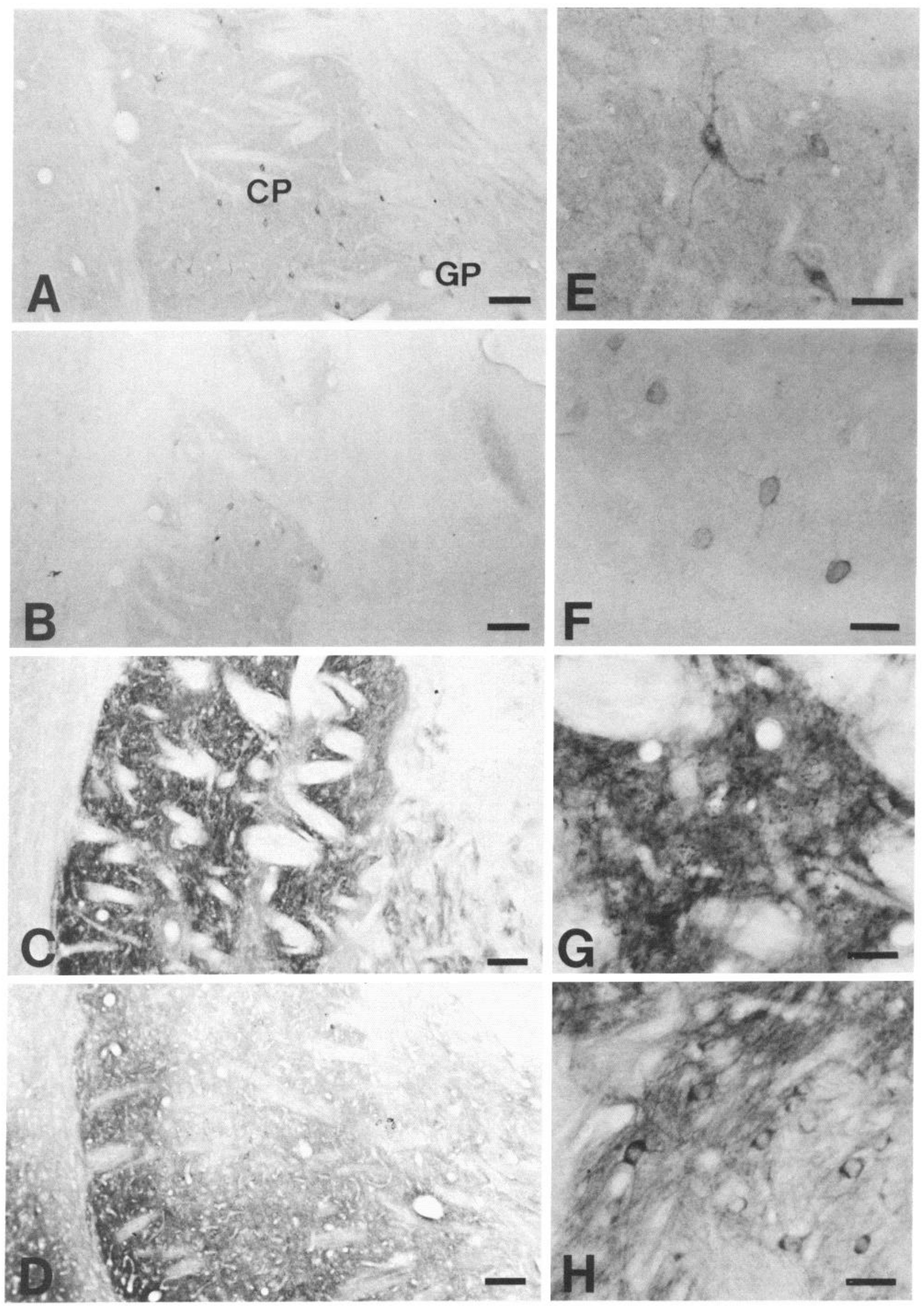




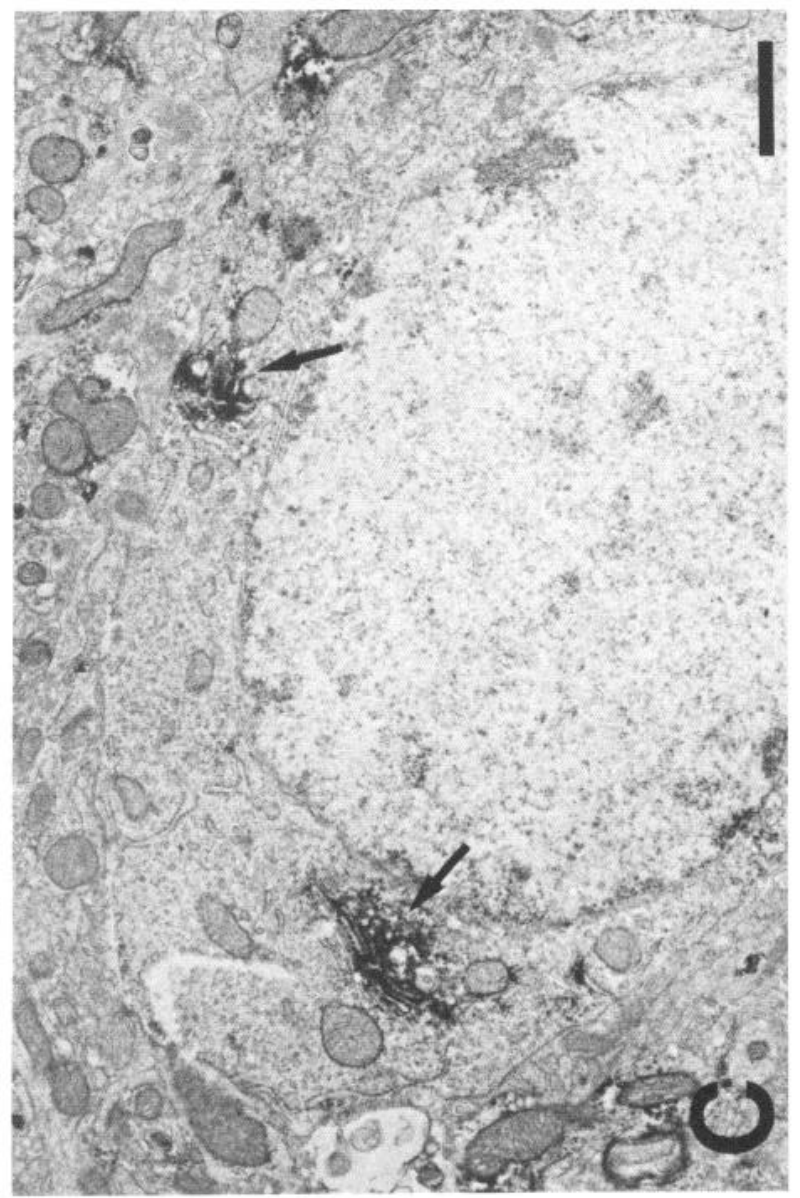

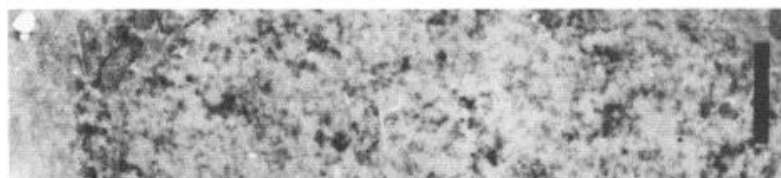

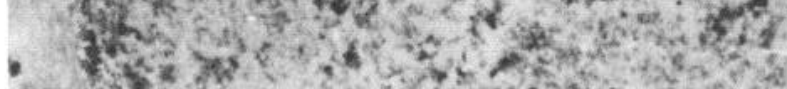

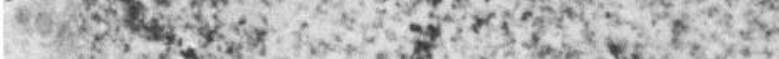
$4 x^{2}$, w in th th

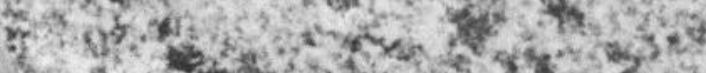

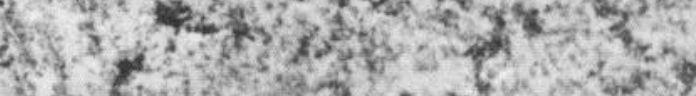

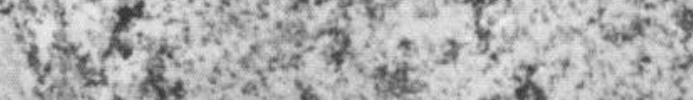
There $x^{2}+2$

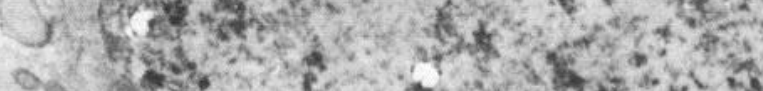

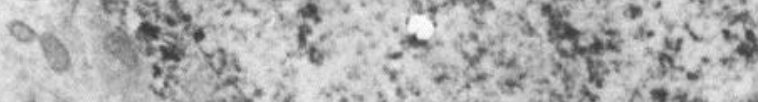

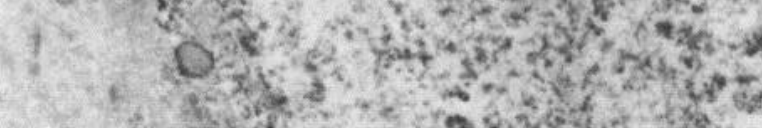
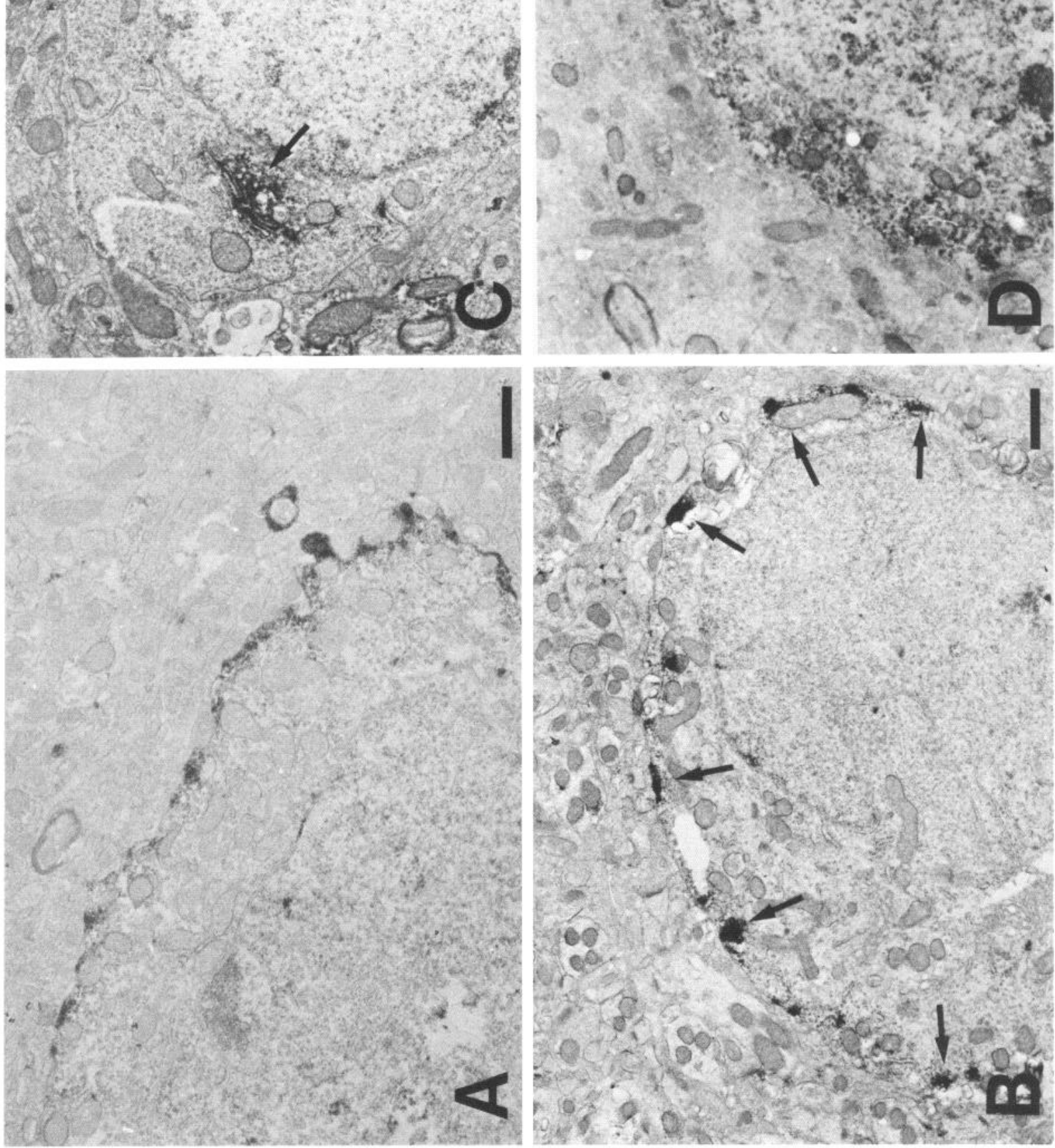
Figure 3. Double staining of $\beta$ II-PKC and $\gamma$-PKC in rat CP. $A$, The $\gamma$-PKC immunoreactivity as demonstrated by the PAP method. $B$, The $\beta$ II-PKC immunoreactivity demonstrated by the indirect FITC-immunofluorescent technique in the same section shown in $A$. The $\gamma$-PKC-immunoreactive neurons were not $\beta$ II-PKC immunoreactive (arrows), and the $\beta$ II-PKC-immunoreactive neurons show no $\gamma$-PKC immunoreactivity (arrowheads). Scale bar, $30 \mu \mathrm{m}$.
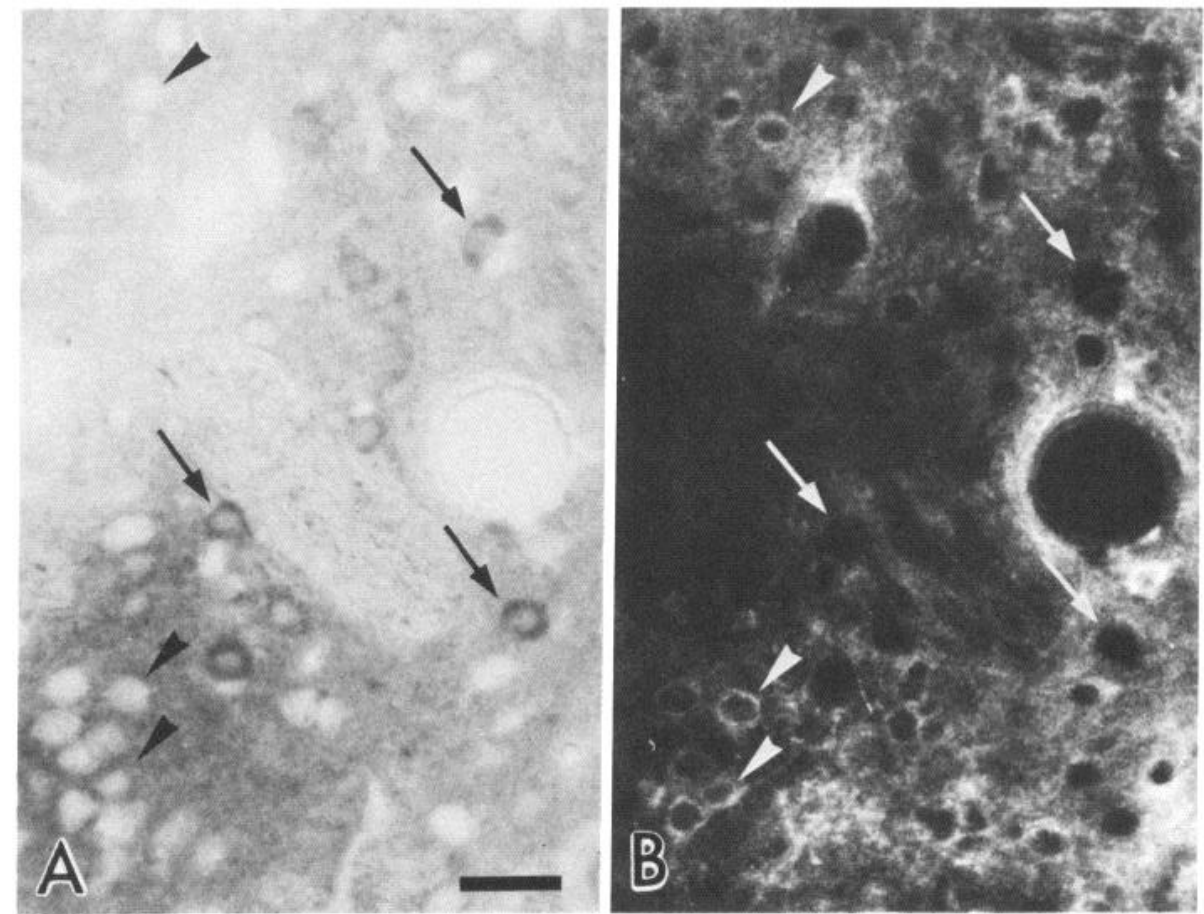

around the concave face (trans region) of the Golgi complex and on its membrane (Fig. 2C). The $\gamma$-PKC immunoreaction was dense throughout the perikaryal cytoplasm, except for the interior of the cell organelles, and light in the nucleoplasm (Fig. 2D).

\section{Colocalization of the PKC subspecies and various neurotransmitter markers}

Double staining between 4 subspecies of PKC showed that no PKC subspecies coexisted with other PKC subspecies in the $\mathrm{CP}$ and GP. Even $\beta$ II-PKC- and $\gamma$-PKC-immunoreactive neurons, which were similar in size (approximately $20 \mu \mathrm{m}$ in diameter), did not overlap (Fig. 3 ). The $\beta$ II-PKC-immunoreactive neurons showed no $\gamma$-PKC immunoreactivity, and the $\gamma$-PKC-immunoreactive ones were not $\beta$ II-PKC immunoreactive.

Approximately $85 \%$ of the large $\alpha$-PKC-immunoreactive neurons showed CAT immunoreactivity, and all these immunoreactive neurons were $\alpha$-PKC immunoreactive (Fig. 4, Table 1). The $\beta \mathrm{I}-, \beta \mathrm{II}-$, and $\gamma$-PKC-immunoreactive neurons contained no CAT immunoreactivity.

The antibody against GABA intensely labeled the scattered medium-sized neurons in the untreated rats. After colchicine treatment, additional medium-sized neurons were labeled with the same antibody. The CP contained 2 types of GABA-immunoreactive neurons that differed in size and staining properties: (1) neurons (25-28 $\mu \mathrm{m}$ in diameter) that exhibited intense
Figure 4. Double staining of $\alpha$-PKC and CAT in rat CP. $A$, The $\alpha$-PKC immunoreactivity as demonstrated by the PAP technique. $B$, The CAT immunoreactivity as demonstrated by the indirect FITC-immunofluorescent technique in the same section shown in $A$. Most of the $\alpha$-PKC-immunopositive neurons (black arrows) show CAT immunoreactivity (white arrows). Scale bar, $30 \mu \mathrm{m}$.

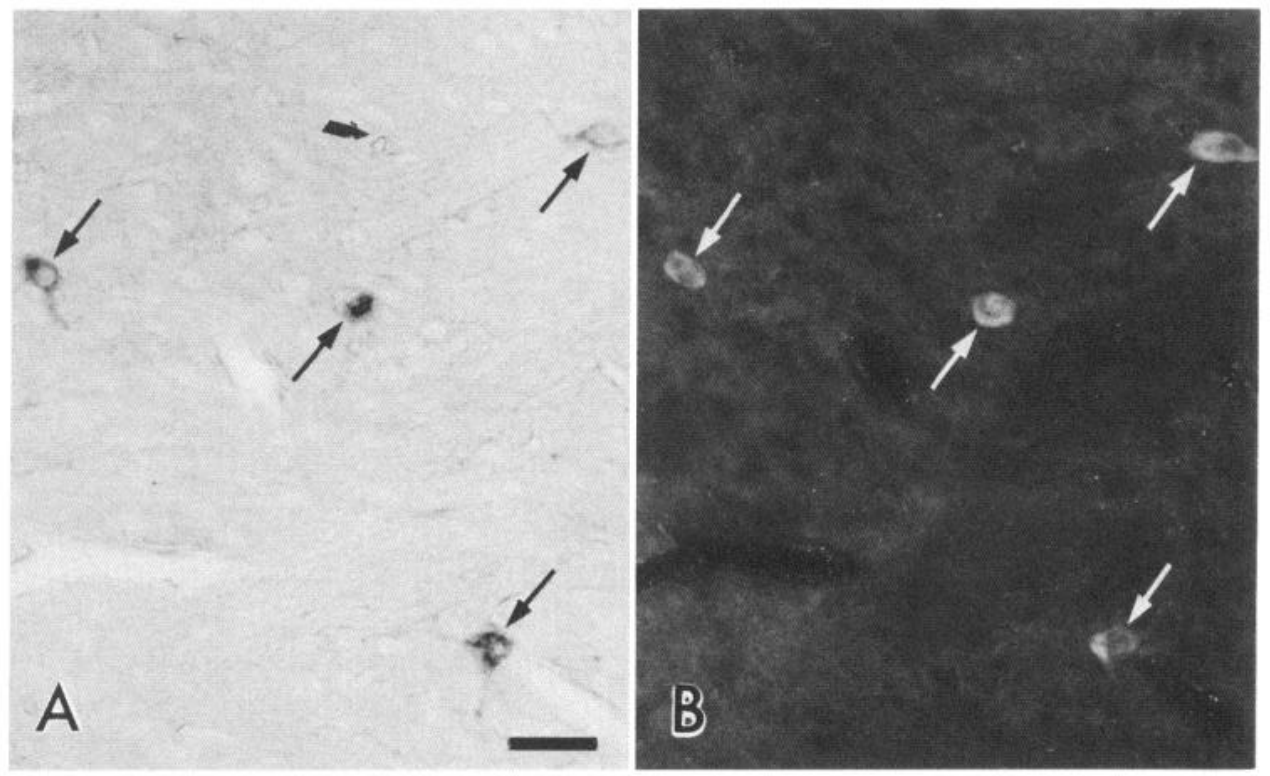



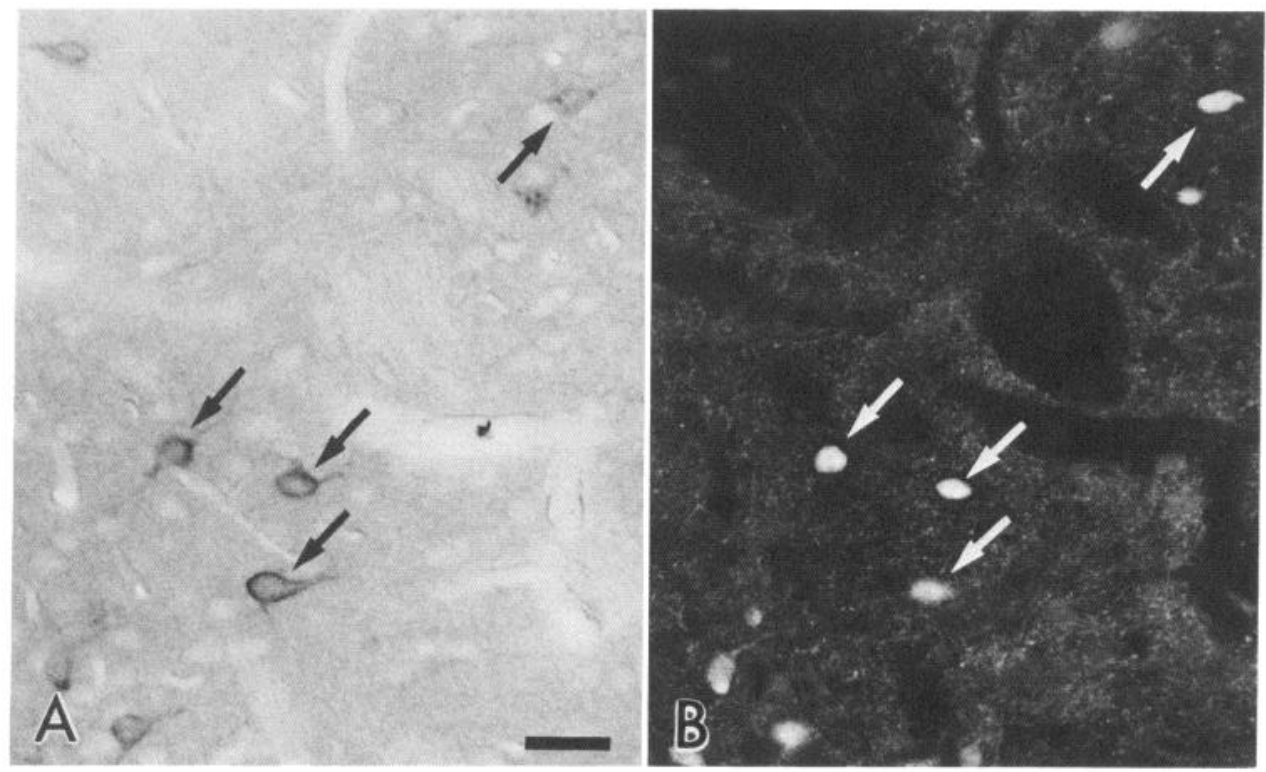

Figure 5. Double labeling of $\beta \mathrm{I}-\mathrm{PKC}$ and GABA immunoreactivity in rat $\mathrm{CP}$. $A$, The $\beta \mathrm{I}-\mathrm{PKC}$ immunoreactivity as demonstrated by the PAP method. $B$, The GABA immunoreactivity as demonstrated by the indirect FITC-immunofluorescent method in the same section shown in $A$. Most of the $\beta$ I-PKCimmunoreactive neurons (black arrows) exhibit GABA immunoreactivity (white arrows). Scale bar, $30 \mu \mathrm{m}$.

GABA immunoreactivity in the rat striatum treated with or without colchicine, and (2) neurons (approximately $20 \mu \mathrm{m}$ in diameter) that displayed light GABA immunoreactivity only in the striatum treated with colchicine. Approximately $80 \%$ of the intensely GABA-immunoreactive neurons showed the $\beta \mathrm{I}-\mathrm{PKC}$ immunoreactivity in the untreated rat striatum (Fig. 5, Table 1). More than $90 \%$ of the lightly GABA-immunoreactive neurons exhibited immunoreactivity for $\beta \mathrm{II}-\mathrm{PKC}$ in the striatum treated with colchicine (Fig. 6, Table 1). Only a small population (less than 10\%) of the $\gamma$-PKC-immunoreactive neurons displayed a weak immunoreaction for GABA (Fig. 7, Table 1) in the colchicine-treated but not untreated rats.

\section{PKC subspecies in the substantia nigra}

Immunoreactivity of $\alpha$-PKC in the neuropils and in the large neurons mostly localized in the pars compacta was evident (Fig. $8 A)$. The $\beta \mathrm{I}-\mathrm{PKC}$-immunoreactive neurons were scattered throughout the pars compacta and pars reticulata of the $\mathrm{SN}$, but the neuropils showed very weak immunoreactivity (Fig. $8 B$ ). Both the $\alpha$-and $\beta$ I-PKC immunoreactivities were localized along the cellular membrane. In contrast to the $\alpha$ - and $\beta \mathrm{I}-\mathrm{PKC}$, both the $\beta$ II- and the $\gamma$-PKC were abundantly present in the neuropils, which showed more intense immunoreactivity in the medial than in the lateral part of the pars reticulata, whereas neither labeled the neuronal perikarya (Fig. $8 C, D$ ).

Double-labeling methods revealed that more than $90 \%$ of the $\alpha$-PKC-immunoreactive cell bodies in the $\mathrm{SN}$ were immunoreactive for TH (Fig. 9, Table 1), while neither GABA nor TH was colocalized in the $\beta \mathrm{I}-\mathrm{PKC}$-immunoreactive cells (data not shown).

To elucidate the neuronal connection between the immunoreactive neurons in the $\mathrm{CP}$ and the immunoreactive neuropils in the $\mathrm{SN}$, we examined the change of immunoreactivities for the PKC subspecies and neurotransmitter markers in the SN after unilateral injection of kainic acid into the head of the CP. The $\beta$ II- and $\gamma$-PKC immunoreactivities showed marked decreases on the injected side. The $\beta$ II-PKC immunoreaction in the neuropils almost disappeared, while the $\gamma$-PKC immunoreaction in the neuropils partly disappeared but remained in the medial part of the pars reticulata. For $\alpha$ - and $\beta \mathrm{I}-\mathrm{PKC}$, no significant difference of the immunoreactivity was seen between control and injected sides (Fig. 10).

\section{Control study}

Immunostaining for the PKC subspecies and neurotransmitter markers was abolished by substitution of preimmunized rabbit or mouse serum for each primary antiserum or by preadsorption of the primary antiserum with the respective antigen.

For double staining, sections that served as controls for the second step consistently failed to display FITC-labeled neurons, demonstrating (1) that the residual HRP from the first labeling step fails to react with the second chromogen, and (2) that there is little species cross-reactivity between the pairs of linking antiserum.

Table 1. Candidates for neurotransmitter for the PKC-subspeciespositive cells in the rat striatonigral system

PKC

subspecies

(A) Neurotransmitter $(B) \quad \% A / B^{a} \quad \% B / A^{b}$

Caudate-putamen

$\alpha$-PKC

$\beta I-P K C$

CAT

100.0

85.7

$\beta$ II-PKC

GABA (intrinsic)

78.9

91.3

83.5

$\gamma$-PKC

$-^{c}$

GABA (projecting)

Substantia nigra

$\alpha$-PKC

Dopamine (TH)

84.8

$\beta I-P K C$

Unknown

$-$

Values are the mean of more than 10 different sections from 2 rats.

- Percent is expressed as the number of double-labeled neurons with antibodies for both $A$ and $B$ divided by the number of immunoreactive cells for neurotransmitter marker $B$.

${ }^{\circ}$ Percent is expressed as the number of double-labeled neurons with antibodies for both $A$ and $B$ divided by the number of immunoreactive cells for the PKC subspecies $A$.

'Unexamined. 


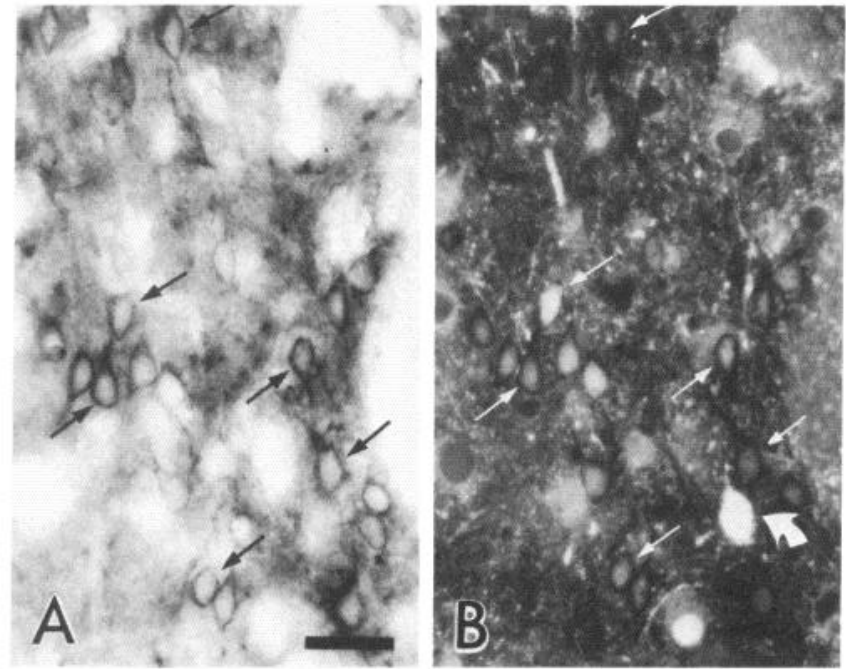

Figure 6. Double staining of $\beta \mathrm{II}-\mathrm{PKC}$ and GABA in rat $\mathrm{CP}$ after colchicine treatment. $A$, The $\beta$ II-PKC immunoreactivity as demonstrated by the PAP method. $B$, The GABA immunoreactivity as demonstrated by the indirect FITC-immunofluorescent technique in the same section shown in $A$. Most of the neurons weakly immunoreactive for GABA (white arrows) show the $\beta$ II-PKC immunoreactivity (black arrows), but the intensely stained GABA-immunoreactive neurons do not contain the $\beta$ II-PKC (thick white arrow). Scale bar, $30 \mu \mathrm{m}$.

\section{Discussion}

We obtained evidence that each subspecies of PKC showed a distinct distribution in the striatum and in the $\mathrm{SN}$ of the rat. Neostriatal neurons can be classified into several populations, based on size and dendritic cytoarchitecture, and also divided into several groups, immunocytochemically, by their neurotransmitter markers. The neuronal network between the neostriatum and the $\mathrm{SN}$ has to some extent been elucidated in immunohistochemical studies and by using tracing techniques. The majority of neostriatal neurons $(70 \%)$ are medium-sized spiny cells that account for most of the neurons projecting to the SN. The medium-sized aspiny type, large spiny type, large aspiny type, and small type make up the remainder (Chang et al., 1982; Heimer et al., 1985).

The localization of $\alpha$-PKC in the CAT-immunoreactive large neurons in the CP suggests that $\alpha$-PKC is involved in certain functions of intrinsic cholinergic neurons. In the SN, the $\alpha$-PKC immunoreactivity was localized in dopaminergic neurons projecting to the striatum. These findings are consistent with reports that CAT could be phosphorylated by PKC (Bruce and Hersh, 1989), and that TH is one of substrates of PKC (Albert et al., 1984; Nishizuka, 1986; Onali and Olianas, 1987). In addition, $\alpha$-PKC may modulate the membrane responsiveness of these neurons, related to the receptors and channels, because $\alpha$-PKC was present predominantly along the perikaryal membrane within both the $\mathrm{CP}$ and the $\mathrm{SN}$.

Resembling $\alpha$-PKC, the $\beta \mathrm{I}-\mathrm{PKC}$ immunoreactivity was predominant in the neuronal cell bodies rather than the neuropils. In the $\mathrm{CP}$, most of the $\beta \mathrm{I}-\mathrm{PKC}$-immunoreactive neurons contained GABA in the rat treated with or without colchicine, observations that suggest that $\beta \mathrm{I}-\mathrm{PKC}$ may play a specific functional role in intrinsic GABAergic neurons. It remains to be clarified which transmitters are contained in the $\beta \mathrm{I}-\mathrm{PKC}$-immunoreactive neurons scattered throughout the pars compacta and reticulata in the $\mathrm{SN}$. The localization of $\beta \mathrm{I}-\mathrm{PKC}$ along the cellular membrane in both regions does suggest that this subspecies is associated with the membrane responsiveness in the intrinsic GABAergic neurons.

In contrast, the $\beta$ II- and $\gamma$-PKC immunoreactivities showed different characteristics from $\alpha$ - and $\beta$ I-PKC: (1) The $\beta$ II- and $\gamma$-PKC immunoreactivities were distributed not only in the numerous medium-sized neurons, but also in the neuropils in the $\mathrm{CP}$ and GP; (2) in the $\mathrm{SN}$, the neuropils displayed intense immunoreactivity for $\beta \mathrm{II}$ - and $\gamma$-PKC, but there were no immunoreactive cell bodies; and (3) the immunoreactivity in the neuropils of the ipsilateral $\mathrm{SN}$ was decreased in the rats treated with kainic acid, a compound that selectively destroys neuronal cell bodies. These findings suggest that the $\beta \mathrm{II}-$ and $\gamma$-PKC immunoreactivities in the $\mathrm{SN}$ are present in the afferent fibers from the striatum to the SN. Worley et al. (1986) reported that phorbol ester binding sites of the $\mathrm{SN}$ decreased in the rat caudate
Figure 7. Double staining of $\gamma$-PKC and $\mathrm{GABA}$ in rat $\mathrm{CP}$ after colchicine treatment. $A$, The $\gamma$-PKC immunoreactivity as demonstrated by the PAP method. $B$, The GABA immunoreactivity as demonstrated by the indirect FITC-immunofluorescent method in the same section shown in $A$. The $\gamma$-PKC-immunoreactive neurons apparently contain no GABA. Scale bar, $30 \mu \mathrm{m}$.
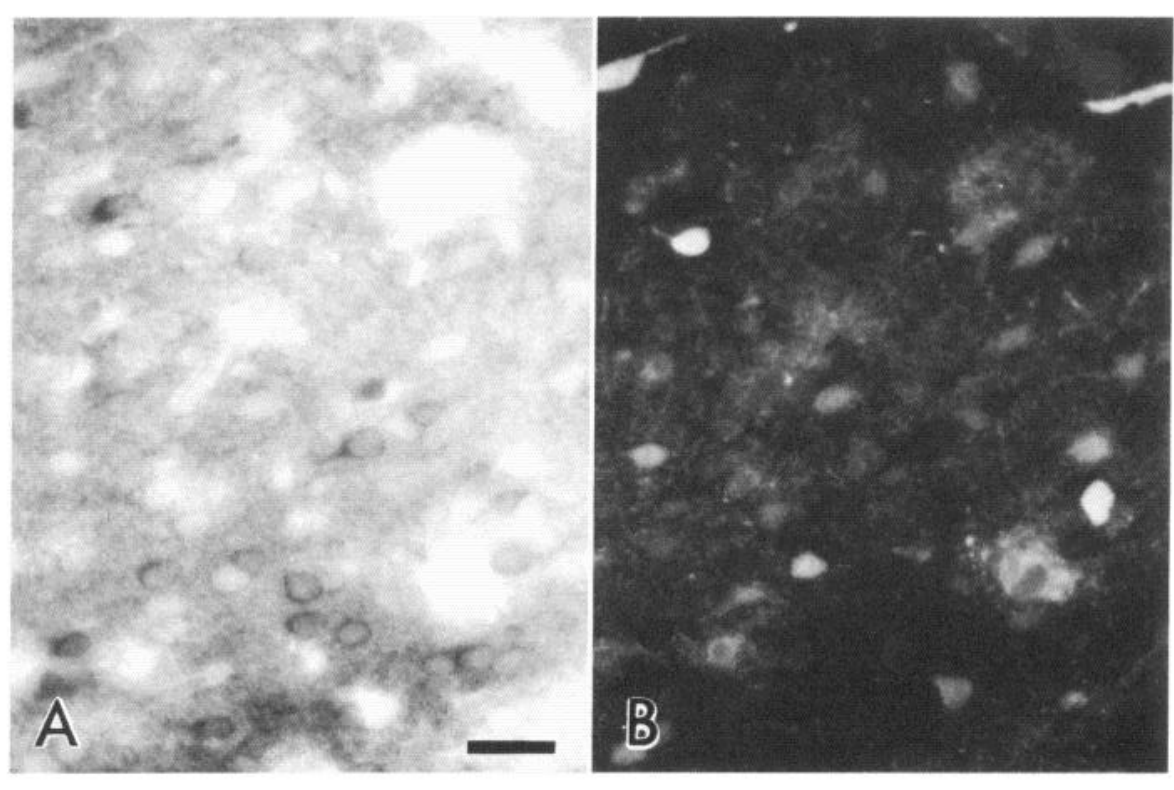


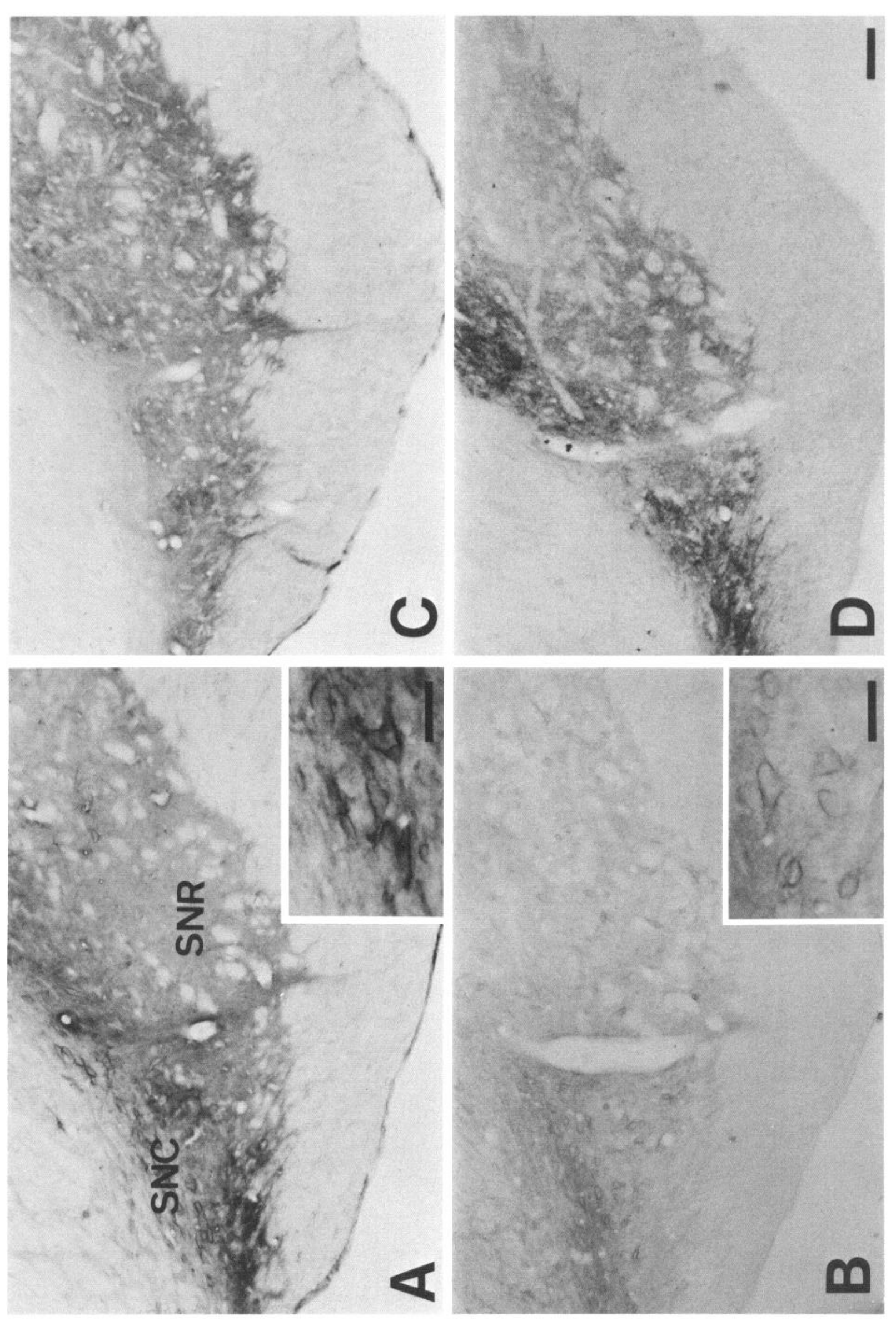

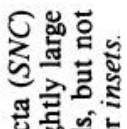

可.

응

i

政

I

西

政

응 8

둥을

응

政

长

들 정

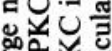

可

을

5ै o

我

ㄷ․들

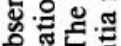

은

总

5 .

论

son

공

西

을

할

政

8 ㅊ. त.

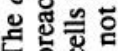

सं

z

a j)

⿷匚⿳丨コ丨

둑

的

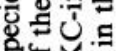

\%

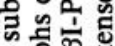

远 20. o 도 过 옹응 8 is बु

记

諾

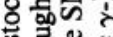

흘을

항

응

항

단흥ㅎㅇ $\infty$ 을

政

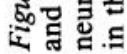


Figure 9. Double staining of $\alpha$-PKC and TH in rat SN. $A$, The $\alpha$-PKC immunoreactivity as demonstrated by the PAP method. $B$, The TH immunoreactivity as demonstrated by the indirect FITC-immunofluorescent technique in the same section shown in $A$. Most of the $\alpha$-PKC-immunoreactive neurons (black arrowheads) show the TH immunoreaction (white arrowheads). Scale bar, $30 \mu \mathrm{m}$
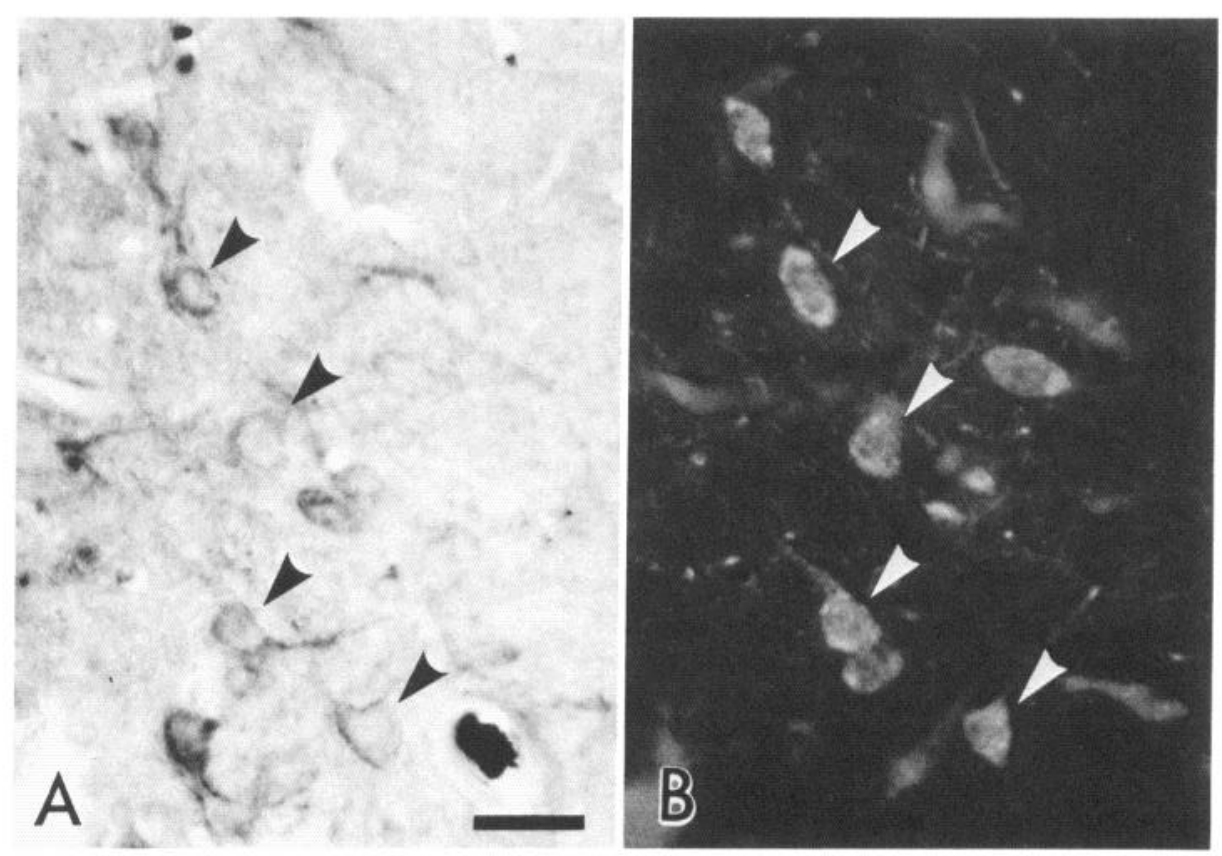

lesioned with quinolinic acid. The present finding suggests that $\beta$ II- or $\gamma$-PKC may participate in the decrease in phorbol ester binding sites. Three neuroactive substances, GABA, substance $\mathrm{P}$, and dynorphin, have been found in the rat striatonigral pathway (Vincent et al., 1982; Bolam et al., 1983; Heimer et al., $1985)$. Double-staining methods revealed that the $\beta$ II-PKC immunoreactivity was colocalized in more than $90 \%$ of the neurons labeled for GABA after colchicine treatment, while $\gamma$-PKC was colocalized only with very few GABAergic neurons. It is likely that $\beta \mathrm{II}-\mathrm{PKC}$ is contained within the striatonigral GABAergic neurons but that most of the $\gamma$-PKC is related to other striatonigral neurons.

There is some evidence that PKC modulates the depolarization-induced release of GABA from the rabbit caudate nucleus (Bartmann et al., 1989) and the KCl-evoked release of GABA from mouse striatal neurons (Weiss et al., 1989). The $\beta \mathrm{I}-\mathrm{PKC}$ in the intrinsic GABAergic neurons might be related to the release of GABA from the striatum. On the other hand, in the $\mathrm{CP}$, the $\beta \mathrm{II}-\mathrm{PKC}$ immunoreaction is remarkably greater than the $\beta \mathrm{I}-\mathrm{PKC}$ immunoreaction in the neuropils. It may be that the release of GABA from the striatal slices includes the release from the axon collaterals of the medium-sized GABAergic neurons, which show $\beta$ II-PKC immunoreactivity. This idea is supported by the finding that the axon collaterals of medium-sized spiny neurons form synaptic junctions with somata, initial segments of axons, dendrites, and dendritic spines of the striatal neurons (Wilson and Groves, 1980). In addition, we found that the activation of PKC by $12-O$-tetradecanoylphorbol-13-acetate enhanced the $\mathrm{KCl}$-evoked release of endogenous GABA from SN slices (K. Taniyama et al., unpublished data). It is suggested that $\beta \mathrm{II}-\mathrm{PKC}$ is involved in the release of GABA in the SN.

There are reports that PKC participates in the release of ACh from striatal slices (Tanaka et al., 1986; Cubeddu et al., 1989) and in the release of dopamine from rat striatal synaptosomes (Chandler and Leslie, 1989). Because $\alpha$-PKC immunoreactivity was present in the cholinergic and dopaminergic cell bodies, it may be involved in the release of these neurotransmitters. Little
$\alpha$-PKC immunoreaction was found in the neuropils of the CP, which contains abundant cholinergic and dopaminergic terminals. It is likely that the $\alpha$-PKC in the nerve terminals is too low in density to be visualized in brain tissues treated with conventional fixatives for immunohistochemistry. It is also possible that yet to be identified PKC subspecies are involved in the release of $\mathrm{ACh}$ and dopamine.

Nishino et al. (1989) found that, in tissues from patients with Parkinson's disease, the phorbol ester binding showed a significant reduction in the SN (by about $60 \%$ ), CP (by about $40 \%$ ), and GP (by about $40 \%$ ). The reduction in the phorbol ester binding in the SN may be attributed to both decreased $\alpha$-PKC in dopaminergic neurons and decreased $\beta$ II-PKC in the GABAergic nerve terminals due to the transsynaptic degeneration of striatal neurons.

In patients with Huntington's disease, there are decreases in several neurotransmitters, including those of GABA, ACh, substance $\mathrm{P}$, and methionine-enkephalin present within the striatonigral pathway. In contrast, dopamine, glutamate, and somatostatin are either not altered or are slightly increased. The concentrations of GABA are decreased $60 \%$ in the $\mathrm{SN}, \mathrm{CP}$, and $\mathrm{GP}$, and there is a $50 \%$ reduction in CAT activity in the $\mathrm{CP}$, while the concentration of substance $P$ is markedly reduced in the striatopallidal (medial pallidum) and striatonigral (pars reticulata) pathways. Pathological changes were predominant in medium-sized spiny neurons, but abnormalities in the mediumsized aspiny and large aspiny neurons were rare (Martin, 1984; Beal and Martin, 1986). The reduction of phorbol ester binding in the striatum and the SN from patients with Huntington's disease may possibly be related to decreases in $\beta \mathrm{II}-$ or $\gamma$-PKC rather than $\beta \mathrm{I}$ - and $\alpha$-PKC.

All these observations suggest that each PKC subspecies has an individual role in the striatal and nigral neurons: $\alpha$-PKC may play a role in regulating the membrane responsiveness in the striatal cholinergic and the nigral dopaminergic neurons, $\beta \mathrm{I}$ PKC may be also involved in the membrane responsiveness in the striatal intrinsic GABAergic neurons, $\beta \mathrm{II}-$ and $\gamma$-PKC are more likely to be related to functions mainly in the striatonigral 

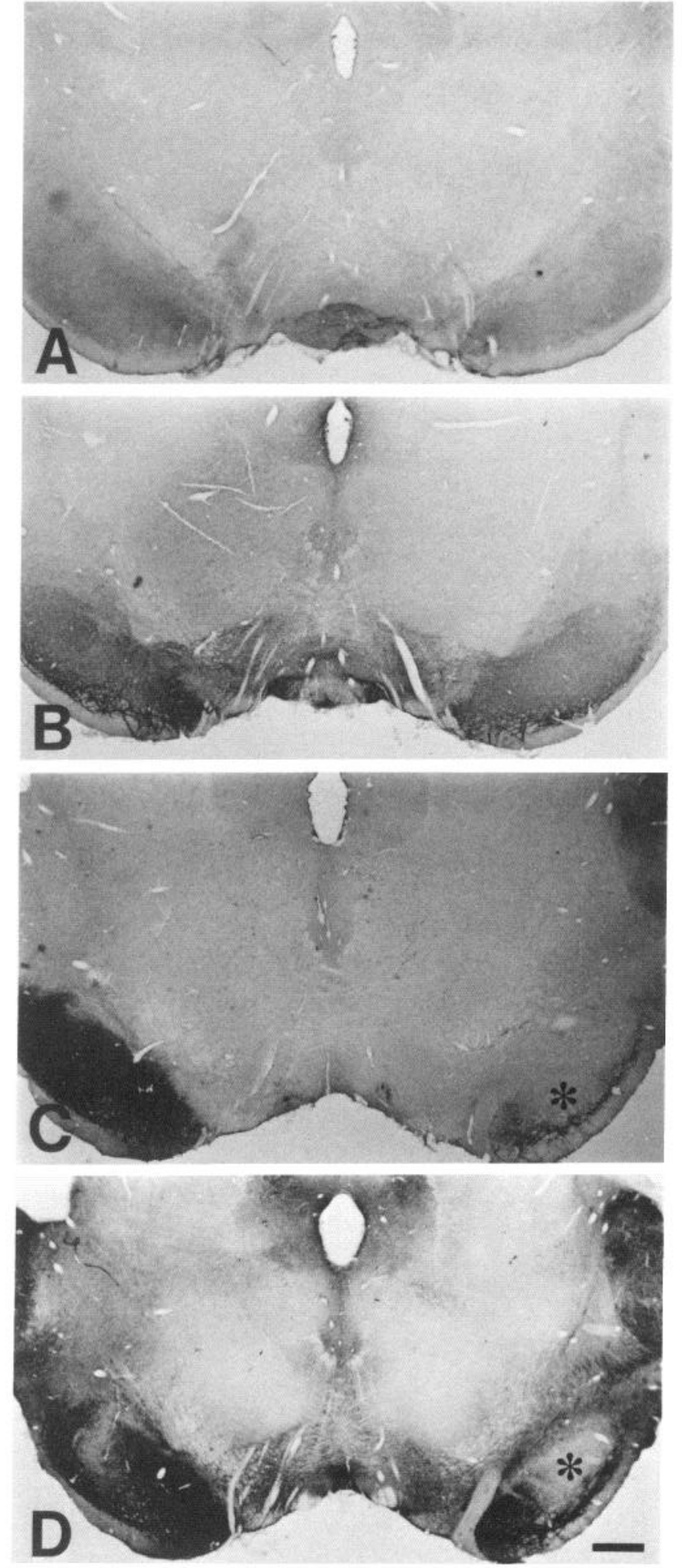

Figure 10. Light microscopic localization of PKC subspecies immunoreactivity in rat $\mathrm{SN}$ after unilateral injection of kainic acid into $\mathrm{CP}$. $A$, The $\alpha$-PKC-immunoreactive neuropils exhibit no difference between injected and control sides. $B$, As is the case with the $\alpha$-PKC, the $\beta \mathrm{I}$ PKC immunoreactivity shows no difference between both sides. $C$, The $\beta$ II-PKC-immunoreactive neuropils are abolished on the injected side (asterisk). D, The $\gamma$-PKC-immunoreactive neuropils show a marked decrease, predominantly in the lateral part of the injection side (asterisk). Scale bar, $500 \mu \mathrm{m}$. projecting neurons, and $\beta \mathrm{II}-\mathrm{PKC}$ may regulate the release of GABA from striatonigral GABAergic neurons.

\section{References}

Albert KA, Helmer-Matyjek E, Nairn AC, Müller TH, Haycock JW, Greene LA, Goldstein M, Greengard P (1984) Calcium/phospholipid-dependent protein kinase (protein kinase $\mathrm{C}$ ) phosphorylates and activates tyrosine hydroxylase. Proc Natl Acad Sci USA 81:77137717.

Bartmann P, Jackisch R, Hertting G, Allgaier C (1989) A role for protein kinase $\mathrm{C}$ in the electrically evoked release of $\left[{ }^{3} \mathrm{H}\right] \gamma$-aminobutyric acid in rabbit caudate nucleus. Naunyn-Schmied Arch Pharmacol 339:302-305.

Beal MF, Martin JB (1986) Neuropeptides in neurological disease. Ann Neurol 20:547-565.

Bolam JP, Somogyi P, Takagi H, Fodor I, Smith AD (1983) Localization of substance P-like immunoreactivity in neurons and nerve terminals in the neostriatum of the rat: a correlated light and electron microscopic study. J Neurocytol 12:325-344.

Bruce G, Hersh LB (1989) The phosphorylation of choline acetyltransferase. Neurochem Res 14:613-620.

Chandler LJ, Leslie SW (1989) Protein kinase C activation enhances $\mathrm{K}^{+}$-stimulated endogenous dopamine release from rat striatal synaptosomes in the absence of an increase in cytosolic $\mathrm{Ca}^{2+}$. J Neurochem 52:1905-1912.

Chang HT, Wilson CJ, Kitai ST (1982) A Golgi study of rat neostriatal neurons: light microscopic analysis. J Comp Neurol 208:107-126.

Cubeddu LX, Lovenberg TW, Hoffman IS, Talmaciu RK (1989) Phorbol esters and $\mathrm{D}_{2}$-dopamine receptors. J Pharmacol Exp Ther 251: 687-693.

Hashimoto T, Ase K, Sawamura S, Kikkawa U, Saito N, Tanaka C, Nishizuka Y (1988) Postnatal development of a brain-specific subspecies of protein kinase $C$ in rat. J Neurosci 8:1678-1683.

Heimer L, Alheid GF, Zaborszky L (1985) Basal ganglia. In: The rat nervous system, Vol 1 (Paxinos G, ed), pp 37-86. New York: Academic.

Hidaka H, Tanaka T, Onoda K, Hagiwara M, Watanabe M, Ohta H, Ito $Y$, Tsurudome M, Yoshida T (1988) Cell type-specific expression of protein kinase $\mathrm{C}$ isozymes in the rabbit cerebellum. J Biol Chem 263:4523-4526.

Hosoda K, Saito N, Kose A, Ito A, Tsujino T, Ogita K, Kikkawa U, Ono Y, Igarashi K, Nishizuka Y, Tanaka C (1989) Immunocytochemical localization of $\beta \mathrm{I}$ subspecies of protein kinase $\mathrm{C}$ in rat brain. Proc Natl Acad Sci USA 86:1393-1397.

Huang FL, Yoshida Y, Nakabayashi H, Huang K-P (1987) Differential distribution of protein kinase $\mathrm{C}$ isozymes in the various regions of brain. J Biol Chem 262:15714-15720.

Huang FL, Yoshida Y, Nakabayashi H, Young WS III, Huang K-P (1988) Immunocytochemical localization of protein kinase $\mathrm{C}$ isozymes in rat brain. J Neurosci 8:4734-4744.

Huang K-P, Nakabayashi H, Huang FL (1986) Isozymic forms of rat brain $\mathrm{Ca}^{2+}$-activated and phospholipid-dependent protein kinase. Proc Natl Acad Sci USA 83:8535-8539.

Huang K-P (1990) Role of protein kinase C in cellular regulation. Biofactors 2:171-178.

Ito A, Saito N, Hirata M, Kose A, Tsujino T, Yoshihara C, Ogita K, Kishimoto A, Nishizuka Y, Tanaka C (1990) Immunocytochemical localization of the $\alpha$ subspecies of protein kinase $\mathrm{C}$ in rat brain. Proc Natl Acad Sci USA 87:3195-3199.

Kikkawa U, Ono Y, Ogita K, Fujii T, Asaoka Y, Sekiguchi K, Kosaka Y, Igarashi K, Nishizuka Y (1987) Identification of the structures of multiple subspecies of protein kinase $C$ expressed in rat brain. FEBS Lett 217:227-231.

Kitamura N, Komure O, Hashimoto T, Kajimoto Y, Nishino N, Tanaka C (1990) Alteration of phosphatidylinositol and adenylate cyclase systems in basal ganglia of patients with Parkinson's disease (PD), Huntington's disease (HD) and multiple system atrophy (MSA). Jpn J Pharmacol [Suppl] 52:113P.

Kitano T, Hashimoto T, Kikkawa U, Ase K, Saito N, Tanaka C, Ichimori Y, Tsukamoto K, Nishizuka Y (1987) Monoclonal antibodies against rat brain protein kinase $\mathrm{C}$ and their application to immunocytochemistry in nervous tissues. J Neurosci 7:1520-1525.

Kose A, Saito N, Ito H, Kikkawa U, Nishizuka Y, Tanaka C (1988) 
Electron microscopic localization of type I protein kinase $\mathrm{C}$ in rat Purkinje cells. J Neurosci 8:4262-4268.

Martin JB (1984) Huntington's disease: new approaches to an old problem. Neurology 34:1059-1072.

Minakuchi R, Takai Y, Yu B, Nishizuka Y (1981) Widespread occurrence of calcium-activated, phospholipid-dependent protein kinase in mammalian tissues. J Biochem 89:1651-1654.

Nishino N, Kitamura N, Nakai T, Hashimoto T, Tanaka C (1989) Phorbol ester binding sites in human brain: characterization, regional distribution, age-correlation, and alterations in Parkinson's disease. J Mol Neurosci 1:19-26.

Nishizuka $Y$ (1984) The role of protein kinase $C$ in cell surface signal transduction and tumour promotion. Nature 308:693--698.

Nishizuka Y (1986) Studies and perspectives of protein kinase C. Science 233:305-312.

Nishizuka Y (1988) The molecular heterogeneity of protein kinase C and its implications for cellular regulation. Nature 334:661-665.

Ohno S, Kawasaki H, Imajoh S, Suzuki K, Inagaki M, Yokokura H, Sakoh T, Hidaka H (1987) Tissue-specific expression of three distinct types of rabbit protein kinase C. Nature 325:161-166.

Onali P, Olianas MC (1987) Stimulation of dopamine synthesis and activation of tyrosine hydroxylase by phorbol diesters in rat striatum. Life Sci 40:1219-1228

Ono Y, Kikkawa U, Ogita K, Fujii T, Kurokawa T, Asaoka Y, Sekiguchi K, Ase K, Igarashi K, Nishizuka Y (1987) Expression and properties of two types of protein kinase $C$ determined by alternative splicing from a single gene. Science 236:1116-1120.

Saito N, Tanaka C (1986) Immunohistochemical demonstration of GABA containing neurons in the guinea pig ileum using purified GABA antiserum. Brain Res 376:78-84.
Saito N, Kikkawa U, Nishizuka Y, Tanaka C (1988) Distribution of protein kinase C-like immunoreactive neurons in rat brain. J Neurosci 8:369-382.

Saito N, Kose A, Ito A, Hosoda K, Mori M, Hirata M, Ogita K, Kikkawa U, Ono Y, Igarashi K, Nishizuka Y, Tanaka C (1989) Immunocytochemical localization of $\beta$ II subspecies of protein kinase $C$ in rat brain. Proc Natl Acad Sci USA 86:3409-3413.

Sakaue M, Kumoi K, Saito N, Tanaka C (1989) Immunocytochemical demonstration of GABA and aspartate-containing neurons in rat inferior olivary nuclei. Biomed Res [Suppl 3] 10:287-295.

Tanaka C, Fujiwara H, Fujii Y (1986) Acetylcholine release from guinea pig caudate slices evoked by phorbol ester and calcium. FEBS Lett 195:129-134.

Vincent S, Hökfelt T, Christensson I, Terenius L (1982) Immunohistochemical evidence for a dynorphin immunorcactive striato-nigral pathway. Eur J Pharmacol 85:251-252.

Weiss S, Ellis J, Hendley DD, Lenox RH (1989) Translocation and activation of protein kinase $C$ in striatal neurons in primary culture: relationship to phorbol dibutyrate actions on the inositol phosphate generating system and neurotransmitter release. J Neurochem 52 : $530-536$.

Wilson CJ, Groves PM (1980) Fine structure and synaptic connections of the common spiny neuron of the rat neostriatum: a study employing intracellular injection of horseradish peroxidase. J Comp Neurol 194: 599-615.

Worley PF, Baraban JM, Snyder SH (1986) Heterogeneous localization of protein kinase $\mathrm{C}$ in rat brain: autoradiographic analysis of phorbol ester receptor binding. J Neurosci 6:199-207. 